\title{
Th1 and Th17 but no Th2-related cytokine spectrum in the cerebrospinal fluid of children with Borrelia-related facial nerve palsy
}

\author{
Zuzana Liba", Jana Kayserova ${ }^{2}$ and Vladimir Komarek ${ }^{1}$
}

\begin{abstract}
Background: Chemokines and cytokines in cerebrospinal fluid (CSF) and serum have been extensively studied in adults with neuroborreliosis (NB), whereas there are limited data about the pediatric population. In adults, T helper type 1 (Th1) and Th17-related cytokines were observed during acute NB. In children, the Th2 response is thought to moderate the disease course. The aim of this study was to determine the chemokine-cytokine profile in children with acute NB displaying Borrelia-related peripheral facial nerve palsy (PFNP).

Methods: Luminex multiple bead technology was used for the detection of twelve cytokines and chemokines in the CSF and serum of three groups: 1) children with Borrelia-related PFNP (BPFNP); 2) children with non-borrelial "idiopathic" PFNP (NI PFNP); and 3) age-related controls.

Results: In B BFNP, cytokines-chemokines related to a non-specific pro-inflammatory activity and specific Th1/Th17 responses were detected in CSF, and elevated IL-7 and IL-10 levels were observed in serum and CSF compared to NIPFNP and to controls. In NIPFNP, CSF findings were similar to controls; however, higher levels of IL-7 and MCP-1 were observed in serum. Higher IL-8, IL-15 and MCP-1 levels were detected in CSF compared to serum in all groups. MCP-1 and IL-8 levels in CSF were strikingly higher in BPFNP Compared to the other two groups, while IL-15 levels in CSF showed no difference. In addition, in controls, increased IL-4 level was found in CSF compared to serum.

Conclusion: The chemokine-cytokine profile in the CSF of children with acute NB was similar to previous studies in adults. Our data suggests that higher levels of IL-4, IL-15 and MCP-1 levels in CSF compared to serum in controls might represent a potentially protective cytokine milieu in the CNS compartment.
\end{abstract}

Keywords: Children, Facial palsy, Neuroborreliosis, Cerebrospinal fluid, Cytokine, IL-4, IL-15, IL-17

\section{Background}

Neuroborreliosis (NB) is characterized by neurologic involvement during infection with the complex of Borrelia burgdorferi sensu lato $(B b)$ [1]. The clinical manifestation and clinical course of NB is different in children compared to adults [2]. Cranial neuropathy including the facial nerve is the most common manifestation of acute NB in the pediatric population $[3,4]$. The disease course seems to have a better prognosis and chronic NB is rarely observed in children [4].

\footnotetext{
* Correspondence: zuzana.liba@gmail.com

'Department of Pediatric Neurology, University Hospital Motol, Prague, Czech Republic

Full list of author information is available at the end of the article
}

Borrelia-species are facultative intracellular pathogens that are able to survive in the extracellular matrix [1]. All of the known types of immune response ( $\mathrm{T}$ helper (Th) type 1, Th2 and Th17) as well as regulatory mechanisms are involved in $B b$-induced inflammation [5-9]. Increased $\mathrm{CD}^{+}$interferon gamma (IFN- $\gamma$ ) -producing $\mathrm{T}$ cells were described in NB patients [7]. A strong Th1 inflammatory response, represented by production of IFN- $\gamma$ and tumor necrosis factor alpha (TNF- $\alpha$ ), is required for successful $B b$ eradication $[7,8]$. The Th2 immune response, represented by interleukin (IL)-4 and IL-13, may inhibit the potentially harmful effect of the Th1 response [5]. The benign course of NB in children was associated with elevated secretion of interleukin (IL)-4 in the CSF [8-10]. Recently, the Th17 response, represented by IL-17, has been

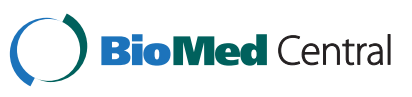


observed in some cases of confirmed NB, and IL-17 has been suggested as an important mediator of $B b$-induced immunopathology [11,12]. Increased levels of IL-17 were associated with more pronounced pleocytosis and fatigue in adults but not in children [12].

Cytokines have been extensively studied in adults with $\mathrm{NB}$, whereas there are limited data about the pediatric population [6-11]. The aim of this study was to determine the concentrations of chemokines and cytokines (IFN- $\gamma$, IL-2, IL-4, IL-6, IL-7, IL-8, IL-10, IL-13, IL-15, IL-17, MCP-1 and TNF- $\alpha$ ) in CSF and serum of children with Borrelia-related peripheral facial nerve palsy (PFNP) and to compare these data to children with non-borrelial "idiopathic" PFNP and to age-related controls.

\section{Methods}

\section{Subjects}

Thirty-four children with PFNP treated in the Pediatric Neurology Department, University Hospital Motol, Prague, Czech Republic, were included in the study: 20 children with Borrelia-related PFNP $\left(\mathrm{B}_{\mathrm{PFNP}}\right)$ and 14 children with non-inflammatory "idiopathic" PFNP $\left(\mathrm{NI}_{\mathrm{PFNP}}\right)$. All underwent lumbar puncture and serum withdrawal within five days of PFNP diagnosis (median time three days). All children with proven Borrelia-related facial palsy (see below) were treated with ceftriaxone intravenously (21 days, dose $50 \mathrm{mg} / \mathrm{kg} /$ day) [13]. A control group consisting of 23 age-matched children without PFNP, underwent lumbar puncture and serum withdrawal to exclude neuroinfection. Their symptoms were as follows: headache $(n=12)$, anomaly of dura mater $(\mathrm{n}=2)$, abnormal gait $(\mathrm{n}=1)$, vertebral algic syndrome $(n=1)$, orthostatic collapses $(n=1)$, paresthesia $(\mathrm{n}=1)$, visual impairment $(\mathrm{n}=1)$, sphincter problems $(\mathrm{n}=1)$, sleep myoclonus $(\mathrm{n}=1)$, macrocephaly $(\mathrm{n}=1)$, cerebral palsy $(n=1)$. Objective neurological examination, magnetic resonance (MR) imaging and analysis of CSF and serum showed normal results. For group specifications, see Table 1 . The project was approved by the local Ethical Committee and the University Hospital Motol. Informed consent was obtained from parents of all participants.

\section{Routine analysis and diagnostic process}

CSF and serum were routinely examined in the Laboratory of CSF and in the Laboratory of Microbiology in University Hospital Motol. Cell count, protein level, blood-brain barrier (BBB) status, level of immunoglobulin (Ig) G and IgM were evaluated by standard methods. Pleocytosis was defined as total cell count in CSF $>5 \times 10^{6} / \mathrm{L}$. Status of the BBB was determined by albumin ratio Qalb (Qalb = albumin CSF $[\mathrm{g} / \mathrm{L}] /$ albumin serum $\times 10^{3}[\mathrm{~g} / \mathrm{L}]$; Qalb $>5$ determinates BBB deficiency [14]. The normal IgG level determined in the laboratory was $<0.040 \mathrm{~g} / \mathrm{L}$ in CSF and $3.6-11.9 \mathrm{~g} / \mathrm{L}$ in serum. The normal IgM level was $<0.0012 \mathrm{~g} / \mathrm{L}$ in CSF and $0.6-2.5 \mathrm{~g} / \mathrm{L}$ in serum $[14,15]$. In cases of elevated levels of Ig in CSF, Reiber's formula for intrathecal production of IgG and/or IgM was used [16].

The presence of antibodies against $B b$ was determined by using the enzyme-linked-immunosorbentassay (ELISA) and western-blot (WB) for verification. Furthermore, CSF and serum were tested for the presence of specific antibodies against herpes-simplex virus, varicella-zoster virus, Epstein-Barr virus and the virus of tick-born encephalitis.

Borrelia-related peripheral facial nerve palsy $\left(\mathrm{B}_{\mathrm{PFNP}}\right)$ was diagnosed when there was pleocytosis in CSF and Borrelia-specific antibodies were present [12]. In case of PFNP with normal CSF and no specific antibody production, high-resolution computer tomography of pyramidal bones (HRCT) and MR imaging of the brain were performed. Idiopathic PFPN was diagnosed when all these were normal ( $\left.\mathrm{NI}_{\mathrm{PFNP}}\right)$.

Table 1 Characteristics of the patient groups

\begin{tabular}{cccc}
\hline & B PFNP $_{(\mathbf{n}=\mathbf{2 0})}$ & $\mathbf{N l}_{\text {PFNP }}(\mathbf{n}=\mathbf{1 4})$ & Controls ( $\mathbf{n}=\mathbf{2 3})$ \\
\hline Age & 7.75 & 9.6 & 10 \\
(median, range) & $(4.2-14)$ & $(2.2-17)$ & $(0.5-17)$ \\
\hline $\begin{array}{c}\text { Sex ratio } \\
\text { (F:M) }\end{array}$ & $11: 9$ & $7: 7$ & $12: 11$ \\
\hline Leukocytes in CSF & $75^{* * *}$ & 1 & 1 \\
(median, range) $\left[\times 10^{-6} / \mathrm{L}\right]$ & $(6-444)$ & $(0-4)$ & $(0-5)$ \\
\hline Protein in CSF & $0.33^{* * * *}$ & 0.16 & 0.18 \\
$($ median, range) $[\mathrm{g} / \mathrm{L}]$ & $(0.14-0,97)$ & $(0.13-0.34)$ & $(0.12-0.32)$ \\
\hline $\mathbf{Q}_{\text {alb }}$ & $6.0^{* * *}$ & 2.8 & 3.1 \\
\end{tabular}

Borrelia-specific-Ab in CSF

$+$

$\mathrm{B}_{\mathrm{PFNP}}$ children with Borrelia-related facial palsy; $\mathrm{Nl}_{\mathrm{PFNP}}$ children with non-inflammatory idiopathic facial palsy; $\mathrm{Q}_{\mathrm{alb}}=\mathrm{CSF}_{\mathrm{alb}}\left[\mathrm{g} / \mathrm{L} \mathrm{L} / \mathrm{serum}\right.$ alb $[\mathrm{g} / \mathrm{L}] \times 10^{3 .} \mathrm{P}$-values are indicated: *** $p<0,0005 ; * * * * 00,0001$. 
After routine analysis all samples were stored at $-20^{\circ} \mathrm{C}$ and thawed once before chemokine and cytokine analyses.

\section{Chemokine and cytokine detection}

Chemokine and cytokine concentrations in CSF and serum were measured by Luminex multiple bead technology and software according to the manufacturer's instructions (Customized kit, Milliplex Human Cytokine/Chemokine Panel, Millipore Corporation, Billerica, MA, USA). Chemokines IL-8, MCP-1 and cytokines IFN- $\gamma$, IL-2, IL-4, IL-6, IL-7, IL-10, IL-13, IL-15, IL-17 and TNF- $\alpha$ were determined in the three previouslycharacterized groups.

\section{Statistical analysis}

Statistical analyses were performed using GraphPad PRISM, version 6.0. Non-parametric tests were used. Kruskal-Wallis ANOVA was performed to compare multiple study groups, and the Mann-Whitney test was applied as a post-hoc test. Wilcoxon signed ranked test was used for pair analysis of CSF and serum in each group. Correlations between parameters were calculated using Spearman correlation.

\section{Results}

White cell count and protein level in CSF were significantly increased in the $\mathrm{B}_{\mathrm{PFNP}}$ group compared to the other groups (Table 1). Both parameters correlated positively with albumin ratio ( $\mathrm{Q}_{\text {alb, }}$ data not shown).

\section{CSF}

Significantly increased chemokines (MCP-1, IL-8) and cytokines (IL-2, IL-6, IL-7, IL-10, IL-17, IFN- $\gamma$ and TNF$\alpha$ ) were detected in $B_{\text {PFNP }}$ in comparison to CSF levels in the other groups (Figures 1a-f and 2c-f).

\section{Serum}

Increased levels of IL-7 and IL-10 were detected in $\mathrm{B}_{\mathrm{PFNP}}$ compared to serum levels in controls. Serum level of IL-10 in $\mathrm{B}_{\text {PFNP }}$ was also significantly higher than in the $\mathrm{NI}_{\text {PFNP }}$ group. In $\mathrm{NI}_{\mathrm{PFNB}}$ increased levels of MCP-1 and IL-7 were detected in comparison to controls (Figures 1a, e and 2f).

\section{CSF vs. serum}

In all groups, significantly higher levels of IL-8, IL-15 and MCP-1 were detected in CSF compared to serum. Additionally, significantly higher IL-4 level in CSF compared to serum was detected in controls (Figure 2a). IL13 levels did not differ between any groups (Figure 2b).

All children with NB had complete clinical recovery from facial palsy within 21 days of the treatment onset.

\section{Discussion}

The detection of chemokines and cytokines in CSF and serum during acute $\mathrm{NB}$ indicated that inflammation was localized mainly in the CNS compartment with an imprint in peripheral blood. Similar to the published data in adults with an early stage of acute NB, innate pro-inflammatory and specific Th1 and Th17-related cytokines were detected in CSF of children with Borrelia-related PFNP while IL-4/Th2-related cytokine was not $[6-8,12]$.

As was previously described, antigen specific T cells are recruited to the CNS from peripheral blood once they are educated in the lymph nodes $[17,18]$. In accordance with the involvement of adaptive immunity during acute $\mathrm{NB}$, we could observe increased IL-7 level in serum and IL-2 and IL-7 levels in CSF [19]. Increased level of IL-2 in CSF and not in serum in $\mathrm{NB}$ patients reflects a general $\mathrm{T}$ cell activation in the CNS [7].

Moreover, we detected raised levels of IL-10 in CSF and serum of children with acute NB. IL-10 is a cytokine with pleiotropic effects but mainly exerts a strong inhibitory function and prevents tissue damage [20]. $B b$ species are able to induce IL-10 production and thus regulate the immune response to their advantage $[1,19]$. Nevertheless, IL-10 is also able to promote cytotoxic $\mathrm{T}$ cell activity and antibody production [7]. So far, we could only speculate on whether increased IL10 level might help in effective resolution of $B b$-infection.

There is a growing body of evidence that less harmful immune responses are preserved in the CNS and that the intraparenchymal CNS environment is anti-inflammatory $[17,18]$. Certain cytokines, such as IL-4 and IL-15, have been recently studied in the context of neuroinflammation and seem to have a neuroprotective effect in animal models [21-23]. We detected a higher level of IL-15 in the CSF in all groups compared to serum. Additionally, we observed a higher CSF level of IL-4 in comparison to serum in the control group. In contrast, IL4 in CSF of children with Borrelia-related PFNP was not increased compared to serum in our study. That could be caused by the fact that the samples were collected at a very early stage of NB (no more than five days after the neurologic symptoms had occurred). Our findings are in accordance with the previous study, in which early increased secretion of Borreliaspecific IFN- $\gamma$ was observed and subsequent upregulation of IL-4 in CSF was associated with non-chronic NB [8]. The potential regulatory role of IL-4 (Th2) in Th1-mediated pathologies is widely discussed. However, MCP-1 also seems to support a Th2 response [24]. Furthermore, the role of MCP-1 and IL-8 in brain function and development has been recently observed in animal models [25]. 

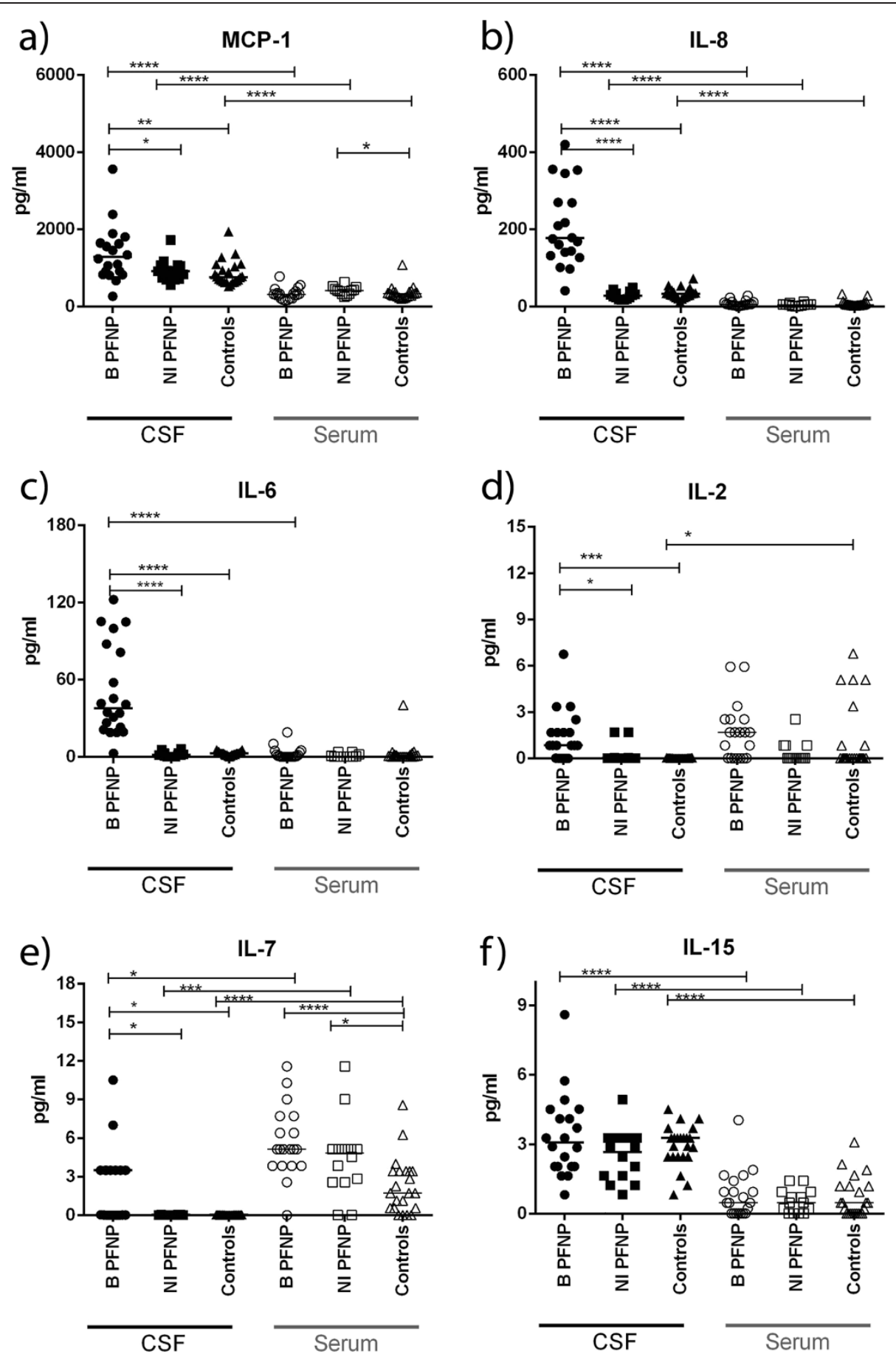

Figure 1 Concentrations of chemokines and cytokines in cerebrospinal fluid (CSF) and serum: a-b) MCP-1 and IL-8 chemokines and c) IL- 6 non-specific pro-inflammatory cytokine were strikingly increased in CSF of BPFNP, d, e) IL-2 and IL-7 cytokines important for T and B cell proliferation and function were increased in CSF of B BFNP (IL-2, IL-7) and in serum of BPFNP and NIPFNP (IL-7), f) IL-15 cytokine was increased in CSF compared to serum in all groups; $B_{\text {PFNP }}(n=20)$ children with Borrelia-related facial palsy; $N I_{\text {PFNP }}(n=14)$ children with non-inflammatory "idiopathic" facial palsy"; Controls $(n=23)$; P-values are indicated: * $p<0,05$; ${ }^{* *} p<0,005$; ** $p<0,0005$; *** $p<0,0001$.

\section{Conclusion}

Despite the slightly different clinical course of acute NB in children, the chemokine and cytokine production in CSF was similar to that of adults. Our data suggests that higher levels of IL-4, IL-15, and MCP-1 in CSF might represent a potentially protective cytokine milieu in the 


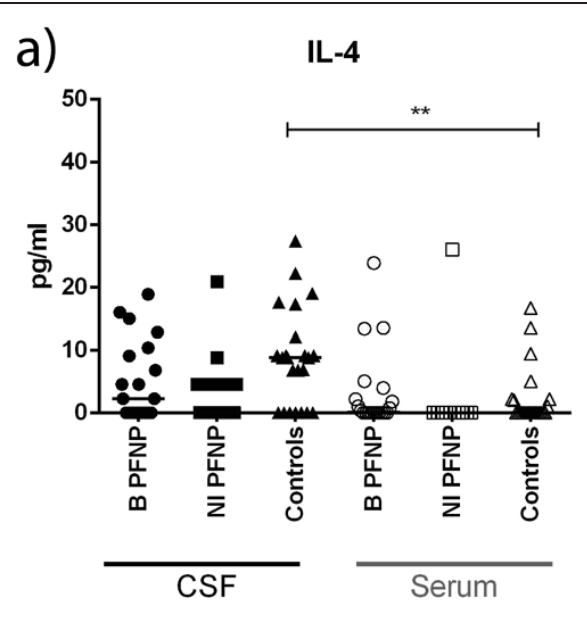
b)
IL-13

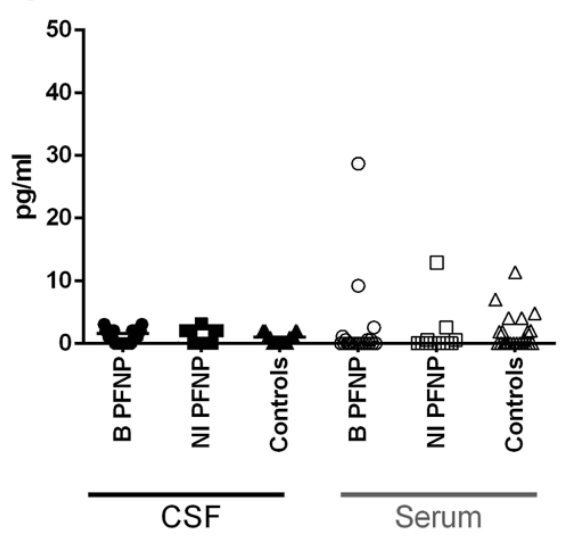

C)

d)
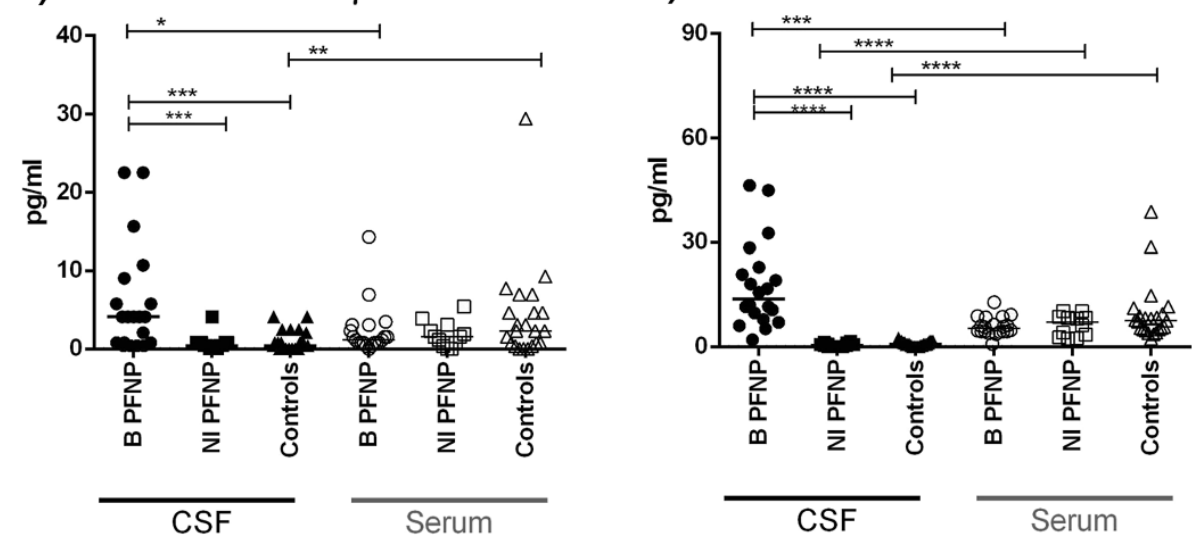

e)

IL-17
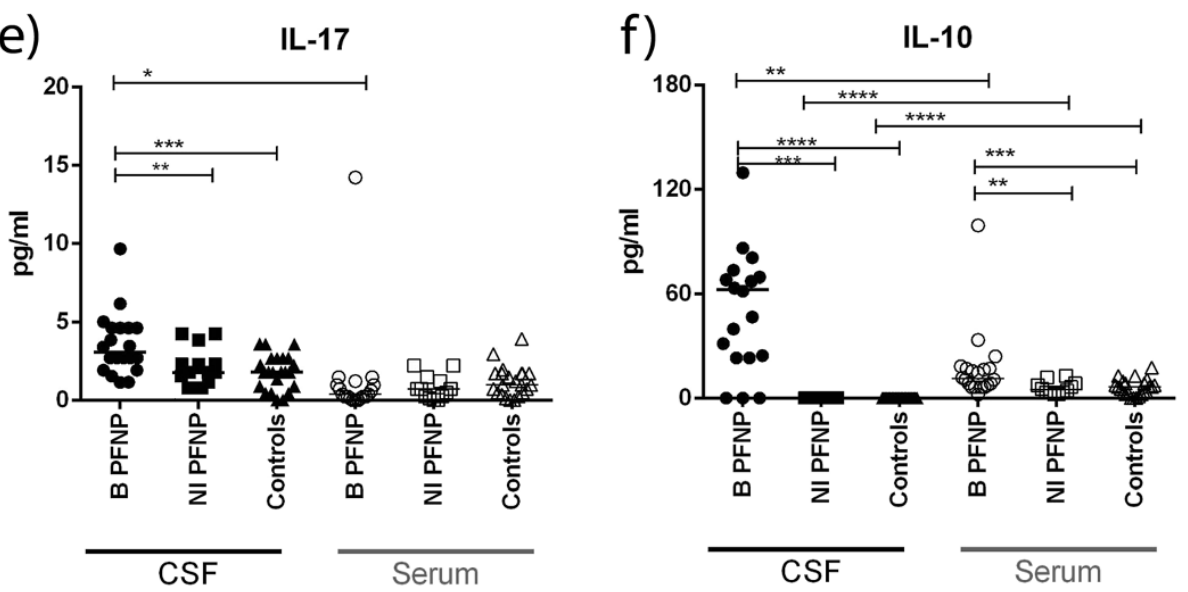

Figure 2 Concentrations of cytokines related to the adaptive immunity in cerebrospinal fluid (CSF) and serum: a-b) IL-4 and IL-13 /Th2 related cytokines - only IL-4 was increased in CSF of controls in comparison to serum, c-f) IFN- $\gamma$ and TNF-a /Th1 related cytokines and IL-17/ Th17 related cytokine were strikingly increased in CSF of BPFNP, $\mathbf{f}$ ) IL-10 regulatory cytokine was strikingly increased in CSF and serum of $B_{\text {PFNP; }}$ $B_{\text {PFNP }}(n=20)$ children with Borrelia-related facial palsy; NI PFNP $(n=14)$ children with non-inflammatory "idiopathic" facial palsy "; Controls $(n=23)$; P-values are indicated: ${ }^{*} p<0,05 ;{ }^{* *} p<0,005$; ** $p<0,0005$; *** $p<0,0001$. 
CNS compartment [20-25]. More studies have to be done to clarify the cytokine environment in the CNS and its role in health and disease.

\section{Competing interests}

The authors declare that they have no competing interests.

\section{Authors' contributions}

ZL is the principal investigator for this project; she conceptualized and designed the project, collected CSF and serum samples, analyzed the results, drafted the manuscript and prepared it for publication. JK performed the laboratory work with sample handling and data acquisition from the Luminex technology and software; she supervised the analysis and intellectual content of the manuscript. VK supervised the clinical data and assisted in the draft of the manuscript. All authors read and approved the final manuscript.

\section{Acknowledgements}

This project was supported by the charity "Movement without Help". We thank Jitka Hanzalova and Devana Dimmerova for routine analysis of the cerebrospinal fluid.

\section{Author details}

'Department of Pediatric Neurology, University Hospital Motol, Prague, Czech Republic. 'Department of Immunology, University Hospital Motol, Prague, Czech Republic.

Received: 19 July 2013 Accepted: 26 September 2013 Published: 4 October 2013

\section{References}

1. Rupprecht TA, Koedel U, Fingerle V, Pfister HW: The pathogenesis of Lyme neuroborreliosis: from infection to inflammation. Mol Med 2008, 14(3-4):205-212.

2. Rizzoli A, Hauffe HC, Carpi G, Vourc'h Gl, Neteler M, Rosà R: Lyme neuroborreliosis in Europe. Eurosurveillance 2011, 16:27.

3. Liba Z, Kayserova J, Komarek V: Frequent incidence of Lyme neuroborreliosis in children in the Czech Republic. Cesk Slov Neurol N 2013, 76/109(1):63-69.

4. López-Alberola RF: Neuroborreliosis and the pediatric population: a review. Rev Neurol 2006, 42(Suppl 3):S91-S96.

5. D'Elios MM, Benagiano M, Della Bella C, Amedei A: T-cell response to bacterial agents. J Infect Dev Ctries 2011, 5(9):640-645.

6. Dennis VA, Dixit S, O'Brien SM, Alvarez X, Pahar B, Philipp MT: Live Borrelia burgdorferi spirochetes elicit inflammatory mediators from human monocytes via Toll-like receptor signaling pathway. Infect Immun 2009, 77(3):1238-1245.

7. Nordberg M, Forsberg P, Johansson A, Nyman D, Jansson C, Ernerudh J, Ekerfelt C: Cytotoxic mechanism may play role in the local immune response in the central nervous system in neuroborreliosis. J Neuroimmunol 2011, 232(1-2):186-193.

8. Widhe $M$, Jarefors $S$, Ekerfelt $C$, Vrethem $M$, Bergstrom $S$, Forsberg $P$, Ernerudh J: Borrelia-specific interferon- $\gamma$ and interleukin-4 secretion in cerebrospinal fluid and blood during Lyme borreliosis in humans: association with clinical outcome. J Infect Dis 2004, 189(10):1881-1891.

9. Sjöwall J, Carlsson A, Vaarala O, Bergström S, Ernerudh J, Forsberg P, Ekerfelt C: Up-regulation of borrelia-specific IL-4 and IFN- $\gamma$ secreting cells in cerebrospinal fluid from children with Lyme neuroborreliosis. Clin Exp Immunol 2005, 141(1):89-98.

10. Skogman BH, Hellberg S, Ekerfelt C, Jenmalm MC, Forsberg P, Ludvigsson J, Bergström S, Ernerudh J: Adaptive and innate immune responsiveness to Borrelia burgdorferi sensu lato in exposed asymptomatic children and children with previous clinical Lyme borreliosis. Clin Dev Immunol 2012, 2012:294587.

11. Oosting $M$, van de Veerdonk FL, Kanneganti TD, Sturm P, Verschueren I, Berende A, van der Meer JW, Kullberg BJ, Netea MG, Joosten LA: Borrelia species induce inflammasome activation and IL-17 production through a caspase-1-dependent mechanism. Eur J Immunol 2011, 41(1):172-181.

12. Henningsson AJ, Tjernberg I, Malmvall BE, Forsberg P, Ernerudh J: Indications of Th1 and Th17 responses in cerebrospinal fluid from patients with Lyme neuroborreliosis: a large retrospective study. J Neuroinflammation 2011, 8:36.

13. Mygland A, Ljøstad U, Fingerle V, Rupprecht T, Schmutzhard E, Steiner I: EFNS guidelines on the diagnosis and management of European Lyme neuroborreliosis. Eur J Neurol 2010, 17(1):8-16.

14. Felgenhauer K: Laboratory diagnosis of neurological diseases. In Clinical laboratory diagnostics - use and assessment of clinical laboratory results. Edited by Thomas L. Frankfurt: TH-Books; 1998:1308-1326.

15. Wildemann B, Oschmann P, Reiber H: Laboratory Diagnosis of Neurological Diseases. Stuttgart: Georg Thieme Verlag; 2010.

16. Deisenhammer F, Bartos A, Egg R, Gilhus NE, Giovannoni G, Rauer S, Sellebjerg F: Routine cerebrospinal fluid (CSF) analysis: report from an EFNS task force. Eur J Neurol 2006, 13(9):913-922.

17. Niederkorn JY: See no evil, hear no evil, do no evil: the lessons of immune privilege. Nat Immunol 2006, 7(4):354-359.

18. Ransohoff RM, Brown MA: Innate immunity in the central nervous system. Clin Invest 2012, 122(4):1164-1171.

19. Unsinger J, McGlynn M, Kasten KR, Hoekzema AS, Watanabe E, Muenzer JT, McDonough JS, Tschoep J, Ferguson TA, McDunn JE, Morre M, Hildeman DA, Caldwell CC, Hotchkiss RS: IL-7 promotes T cell viability, trafficking, and functionality and improves survival in sepsis. $J$ Immunol 2010, 184(7):3768-3779.

20. Gautam A, Dixit S, Philipp MT, Singh SR, Morici LA, Kaushal D, Dennis VA: Interleukin-10 alters effector functions of multiple genes induced by Borrelia burgdorferi in macrophages to regulate Lyme disease inflammation. Infect Immun 2011, 79(12):4876-4892.

21. Wu X, Weihong $P, Y i H$, Hung H, Kastin AJ: Cerebral interleukin 15 shows upregulation and beneficial effects in experimental autoimmune encephalomyelitis. J Neuroimmunol 2010, 223(1-2):65-72.

22. Ponomarev ED, Maresz K, Tan Y, Dittel BN: CNS-derived interleukin-4 is essential for the regulation of autoimmune inflammation and induces a state of alternative activation in microglial cells. J Neurosci 2007, 27(40):10714-10721.

23. Gadani SP, Cronk JC, Norris GT, Kipnis J: IL-4 in the brain: a cytokine to remember. J Immunol 2012, 189(9):4213-4219.

24. Deshmane SL, Kremlev S, Amini S, Sawaya BE: Monocyte chemoattractant protein-1 (MCP-1): an overview. J Interferon Cytokine Res 2009, 29(6):313-326

25. Semple BD, Kossmann T, Morganti-Kossmann MC: Role of chemokines in CNS health and pathology: a focus on the CCL2/CCR2 and CXCL8/CXCR2 networks. J Cereb Blood Flow Metab 2010, 30(3):459-473.

doi:10.1186/2045-8118-10-30

Cite this article as: Liba et al:: Th1 and Th17 but no Th2-related cytokine spectrum in the cerebrospinal fluid of children with Borrelia-related facial nerve palsy. Fluids and Barriers of the CNS 2013 10:30.

\section{Submit your next manuscript to BioMed Central and take full advantage of:}

- Convenient online submission

- Thorough peer review

- No space constraints or color figure charges

- Immediate publication on acceptance

- Inclusion in PubMed, CAS, Scopus and Google Scholar

- Research which is freely available for redistribution 